\title{
High Mobility Group Nucleosomal Binding Domain 2 Protein Protects Bladder Epithelial Cells from Klebsiella pneumoniae Invasion
}

\author{
Yue CaO, Guixia Wu, Bo Fan, Fengjin Zheng, Xiang Gao, Na Liu, Xiaokang Liu, and Ning Huang* \\ Department of Pathophysiology, West China School of Preclinical Sciences and Forensic Medicine, Sichuan University; \\ Chengdu, Sichuan Province 610041, China. \\ Received February 8, 2011; accepted March 22, 2011; published online April 18, 2011
}

\begin{abstract}
Due to the predominance of multiple-antibiotic-resistant Klebsiella pneumoniae strains, the search for new approaches for the prevention of $K$. pneumoniae infections is now under intensive investigation. The objective of the present study was to investigate the effects of high mobility group nucleosomal binding domain 2 (HMGN2) protein, which acts on the bladder epithelial cells T 24, on the invasion of $K$. pneumoniae 03183 and explore its possible mechanisms. Pretreatment with HMGN2 significantly reduced $K$. pneumoniae 03183 uptake by T 24 cells. In T 24 cells, there were no detectable cytotoxic effects of HMGN2 at any concentration between 32 and $256 \mu \mathrm{g} / \mathrm{ml}$ after $2 \mathrm{~h}$ incubation. HMGN2 exhibited no appreciable antibacterial activity against $K$. pneumoniae 03183. Fluorescence microscopy and flow cytometry analysis revealed that HMGN2 blocked $K$. pneumoniae 03183-induced actin polymerization. K. pneumoniae 03183-induced phosphorylation of extracellular signal-regulated kinase (ERK) and cofilin were prevented by pretreatment with HMGN2. These results indicated that pretreatment with HMGN2 inhibited cofilin phosphorylation and then induced actin disruption which may block ERK phosphorylation. These changes led to inhibition of $K$. pneumoniae 03183 invasion of $T 24$ bladder epithelial cells.
\end{abstract}

Key words high mobility group nucleosomal binding domain 2; actin; Klebsiella pneumoniae; extracellular signal-regulated kinase; cofilin

Klebsiella pneumoniae is the second most common cause of Gram-negative urinary tract infection (UTI). Treatment of $K$. pneumoniae infections has become increasingly difficult because of the predominance of multiple-antibiotic-resistant strains. ${ }^{1,2)}$ Increasing antibiotic resistance has led to an urgent need for new therapeutic approaches.

Bacterial invasion to uroepithelial cells is a critical step in the ability of bacteria to cause UTI. Antimicrobial peptides (AMPs) are major components of the innate immune defense system. They are well conserved along evolution, non-toxic and ensure potent defenses against a large number of pathogens. The chromatin-associated high mobility group protein HMGN2 (also known as HMG17) is a member of the HMGN family that binds specifically to nucleosomes ${ }^{3)}$ in the cell nucleus of vertebrate and invertebrate organisms. HMGNs play a role in global DNA repair. ${ }^{4)}$ HMGN proteins serve as a counterbalance to the action of the many ATPdependent chromatin remodeling activities in the nucleus. ${ }^{5)}$ HMGN2 is a major regulator of homeodomain transcription factor activity modulated by Wnt/beta-catenin signaling. ${ }^{6}$ Recently, an additional role for HMGN2 outside the cell nucleus has come into focus. HMGN2 shows potent antimicrobial activity against Escherichia coli ML-35p, Pseudomonas aeruginosa ATCC 27853 and, to some extent, against Candida albicans ATCC 10231. ${ }^{7,8)}$ HMGN2 protein inhibits hepatic B virus (HBV) protein expression and replication in vitro. ${ }^{9)}$ HMGN2 also shows anti-cancer potency in HeLa cells. $^{10)}$

The host cytoskeleton is crucial for mediating pathogen invasion. The internalization mechanism triggered by $K$. pneumoniae 3091 is associated with actin cytoskeleton. ${ }^{11)}$ Association between AMPs and actin has been previously reported in other cell types. ${ }^{12-14)}$ HMGB1 induces rapid and transient changes in actin cytoskeleton reorganization. ${ }^{15)}$ Does the HMGN2 as a member of the HMG family and antimicrobial peptide have the effect on actin cytoskeleton? In this work, using HMGN2 that acts on bladder epithelial cell T24, we investigated the dependence of actin cytoskeleton on the internalization of $K$. pneumoniae 03183 .

\section{MATERIALS AND METHODS}

Bacterial Strains, Cell Lines, and Culture Conditions $K$. pneumoniae 03183 was isolated from a sample of a UTI patient. Bacterial cells were stored at $-70{ }^{\circ} \mathrm{C}$ in LuriaBertani (LB) medium supplemented with $15 \%$ glycerol. Bacterial cells were grown in LB broth at $37^{\circ} \mathrm{C}$ with shaking or solidified with $1.5 \%$ agar. Human epithelial bladder cells T 24 were cultivated in RPMI 1640 medium with 10\% FBS at $37^{\circ} \mathrm{C}$ in an atmosphere of $94 \%$ air $-6 \% \mathrm{CO}_{2}$. All cell culture media and supplements were obtained from GIBCO/ BRL (Gaithersburg, MD, U.S.A.).

Production of HMGN2 Protein To express the recombinant HMGN2 (rHMGN2), the plasmid pET-32a(+)HMGN2 constructed in our previous studies was transformed into the BL21 (DE3) strain of E. coli. The single colony of transformed $E$. coli was picked out and inoculated in a flask containing $500 \mathrm{ml}$ LB medium supplemented with penicillin $(50 \mu \mathrm{g} / \mathrm{ml})$ and cultured at $37^{\circ} \mathrm{C}$ until cell density OD600 reached $0.6-0.7$. The culture was induced by addition of the final concentration of $0.6 \mathrm{~mm}$ IPTG for $3 \mathrm{~h}$ at $37^{\circ} \mathrm{C}$. The induced cultures were washed with phosphate buffered saline (PBS) and the cell lysates were disrupted by sonication (5 times for $30 \mathrm{~s}, 30 \%$ power). After centrifugation, the supernatants were saved. The fusion proteins were purified through HisTrap Chelating HP columns (Pharmacia, U.S.A.) and then cleaved by thrombin digestion. The protein rHMGN2 was obtained by reverse-phase high-performance liquid chromatography (RP-HPLC) purification. Protein concentration was determined by bicinchoninic acid (BCA) Pro- 
tein Assay Kit (Pierce, U.S.A.) and the purity was confirmed by Tricine-sodium dodecyl sulfate-polyacrylamide gel electrophoresis (SDS-PAGE) and Western blotting with a primary antibody mouse anti-His monoclonal antibody (Roche, U.K.).

Invasion Assay For the invasion assay, T 24 cells were seeded at $5 \times 10^{4}$ cells per well in 24 -well plates. Cells were preincubated with substances (rHMGN2 or cytochalasin B or latrunculin B or Y27632 or PD98059) for $2 \mathrm{~h}$ prior to bacterial infection in order to study the cell structures and processes involved in internalization. The monolayers were washed twice with PBS to remove any substances prior to infection with $K$. pneumoniae 03183 . In each well of a 24 -well cell culture cluster, about $5 \times 10^{6}$ mid-log-phase $K$. pneumoniae $03183\left(\mathrm{OD}_{600}=0.4-0.6\right)$ were added to epithelial cells per well. Invasion was allowed to occur for $2 \mathrm{~h}$ at $37^{\circ} \mathrm{C}$ in $5 \% \mathrm{CO}_{2}$. Before a second $2 \mathrm{~h}$ incubation (kill) period under the same conditions but with fresh medium containing $100 \mu \mathrm{g}$ of gentamicin per $\mathrm{ml}$, monolayers were washed once with PBS (pH 7.4). During the kill period, all extracellular $K$. pneumoniae 03183 were killed but the viability of internalized bacteria was not affected. Finally, monolayers were washed twice with PBS and lysed with $0.1 \%$ Triton X-100 and the released intracellular bacteria were enumerated by plate count. The number of intracellular $K$. pneumoniae 03183 after invasion in the presence of an inhibitor was divided by the number of intracellular bacteria in the absence of inhibitors and multiplied by 100 to calculate the percentage of relative invasiveness. All assays were conducted in duplicate and repeated independently at least three times. Cytochalasin B (Cyt B) and PD98059 were purchased from Sigma Chemical (St. Louis, MO, U.S.A.). Latrunculin B (Lat B) and Y27632 were purchased from Calbiochem (San Diego, CA, U.S.A.).

Cell Survival Assay To determine whether rHMGN2 exhibited cytotoxicity, T 24 cells were grown in the presence of different concentrations of rHMGN2 $(0,32,64,128$, $256 \mu \mathrm{g} / \mathrm{ml}$ ) in 96-well cell culture plates for $2 \mathrm{~h}$ (six replicates per treatment group). Wells without rHMGN2 were used as negative controls. After being incubated at $37^{\circ} \mathrm{C}$ in $5 \% \mathrm{CO}_{2}$, the supernatant was discarded and $200 \mu \mathrm{l}$ of $0.5 \mathrm{mg} / \mathrm{ml}$ 3-(4,5-dimethylthiazol-2-yl)-2,5-diphenyl-2Htetrazolium bromide (MTT, Sigma-Aldrich) was added into each well, and then the plate was incubated for another $4 \mathrm{~h}$. The insoluble formazan was collected, dissolved in $200 \mu \mathrm{l} /$ well of dimethylsulfoxide (DMSO) and measured with an enzyme-linked immunosorbent assay (ELISA) reader (Bio-Rad, U.S.A.) at a wavelength of $490 \mathrm{~nm}$.

Extracellular Bacterial Growth Assay and Determination of Antibacterial Activity K. pneumoniae 03183 culture $\left(\mathrm{OD}_{600}=0.6\right)$ was diluted $1: 200$ in fresh LB broth and then dispensed into tubes containing different concentrations of rHMGN2. After thorough mixing, the bacteria suspension was dispensed onto a 96-well plate (six replicates per treatment group). Bacterial growth in each well was monitored spectrophotometrically in a microplate reader at $37^{\circ} \mathrm{C}$ and a wavelength of $600 \mathrm{~nm}$, with readings taken every $30 \mathrm{~min}$ for $4 \mathrm{~h}$.

Bacteria were diluted with PBS solution, $\mathrm{pH} 7.4$, to $1.0 \times 10^{9} / \mathrm{ml}$, added to different concentrations of rHMGN2, and incubated at $37^{\circ} \mathrm{C}$ for 0,2 and $4 \mathrm{~h}$. After incubation and proper dilution, $100 \mu \mathrm{l}$ of each solution was plated onto LB agar to assess bacterial colony forming units (CFUs).

Immunofluorescence Staining Cells were grown in 24well plates at an initial concentration of $5 \times 10^{4}$ cells/well. Substances (rHMGN2 or cytochalasin B) that act on T 24 cells were added to monolayers before the addition of $K$. pneumoniae 03183 for $2 \mathrm{~h}$. At the end of each experiment, the cells were fixed for 10 min with $4 \%$ PFA, permeabilized with PBST for $5 \mathrm{~min}$, preincubated with $1 \%$ bovine serum albumin (BSA)-PBS to prevent nonspecific binding, and incubated with rhodamine-phalloidin (Sigma-Aldrich) diluted in $1 \%$ BSA-PBS at a final concentration of $5 \mu \mathrm{g} / \mathrm{ml}$ for $30 \mathrm{~min}$ in the dark.

Measurement of F-Actin Content by Flow Cytometry Rhodamine-phalloidin (Molecular Probes), which binds directly and specifically to F-actin, was used in a flow cytometric assay to detect F-actin content. rHMGN2 or cytochalasin B acted on T 24 cells before the addition of $K$. pneumoniae 03183 for $2 \mathrm{~h}$. At the end of each experiment, the cells were incubated with rhodamine-phalloidin. After $30 \mathrm{~min}$ at $37^{\circ} \mathrm{C}$, the cells were washed in PBS and resuspended at $10^{6}$ cells $/ \mathrm{ml}$ in PBS for flow cytometry.

Preparation of Cell Lysates T24 cells were seeded at $10^{6}$ cells in $10-\mathrm{cm}$-diameter tissue culture dishes and allowed to grow for $2 \mathrm{~d}$. rHMGN2 was added at various times before the infection process. The monolayers were washed twice with PBS to remove any substances prior to infection. Bacteria were added to the $\mathrm{T} 24$ cells at a multiplicity of infection of approximately $100: 1$. Infection with bacteria was carried out in the same manner as described for the invasion assays. After the infection period, the monolayer was washed twice with ice-cold PBS, pH 7.4. Cells were scraped into $1.5 \mathrm{ml}$ microcentrifuge tubes with disposable cell scrapers. The supernatant was removed after centrifugation at $3000 \mathrm{rpm}$ for $3 \mathrm{~min}$ at $4{ }^{\circ} \mathrm{C}$. The appropriate lysis buffer was added to the pellets, and the samples were spun down at $10000 \mathrm{rpm}$ for $10 \mathrm{~min}$ at $4{ }^{\circ} \mathrm{C}$. The soluble fraction was transferred to a new microcentrifuge tube.

Western Blot Analysis Uninfected and infected cells were pretreated with rHMGN2 for the indicated time points. Proteins were separated by $12 \%$ SDS-PAGE and electrophoretically transferred to polyvinylidene fluoride (PVDF) membranes ( $70 \mathrm{~V}, 1.5 \mathrm{~h})$. Non-specific binding sites of PVDF sheets were then blocked for $1 \mathrm{~h}$ at $37^{\circ} \mathrm{C}$ with $5 \%$ nonfat milk in PBS, pH 7.4, 0.1\% Tween-20. After washing with PBS, $0.1 \%$ Tween-20, the sheets were incubated for $1 \mathrm{~h}$ at room temperature with the primary antibody, including rabbit anti-human extracellular signal-regulated kinase (ERK), cofilin, phospho-cofilin antibodies [Cell Signaling Technology, Danvers, MA, U.S.A. (1:1000)], and mouse anti-human phospho-ERK antibody [BD Bioscience, San Jose, CA, U.S.A. $(1: 1000)]$. Next, the membranes were washed and probed with the secondary antibody coupled to peroxidase for $1 \mathrm{~h}$ at room temperature. The sheets were washed and the proteins were revealed using the ECL detection system according to the manufacturers' instructions.

Statistical Analysis Results were expressed as the mean \pm S.D. and evaluated by Student's $t$-test. A $p$ value of $<0.05$ was considered as significant. 


\section{RESULTS}

Effects of rHMGN2 on Invasion Activity of K. pneumoniae 03183 to T 24 Cells The extent of bacterial invasion was determined on the basis of the ability of bacteria to survive treatment with gentamicin. Gentamicin is a bactericidal antibiotic that is unable to enter eucaryotic cells. Therefore, bacteria adherent to epithelial cells are killed by this drug, while internalized organisms are protected from its effects. $\left.{ }^{16}\right)$ As shown in Fig. 1, the entry of K. pneumoniae 03183 into T 24 cells preconditioned with $\mathrm{rHMGN} 2$ at the dosage of $32-256 \mu \mathrm{g} / \mathrm{ml}$ for $2 \mathrm{~h}$ was inhibited. Because the decrease observed after incubation with $256 \mu \mathrm{g} / \mathrm{ml}$ rHMGN2 did not significantly surpass the effect obtained with $128 \mu \mathrm{g} / \mathrm{ml}$, we deduced the latter concentration as being optimal for causing a considerable reduction.

Effects of rHMGN2 on Cell Survival and Extracellular Bacterial Growth and Bacterial Viability To evaluate the cytotoxic effects of rHMGN2, T 24 cells were grown in the presence of different concentrations of rHMGN2 $(0,32,64$, $128,256 \mu \mathrm{g} / \mathrm{ml})$ in culture plates for $2 \mathrm{~h}$. The MTT assay was used to assess the cytotoxicity of rHMGN2 in T 24 cells. There were no detectable cytotoxic effects of rHMGN2 at any concentration between 0 and $256 \mu \mathrm{g} / \mathrm{ml}$. The survival rates were $99.3 \pm 2.8 \%$ in the $32 \mu \mathrm{g} / \mathrm{ml}, 99.2 \pm 2.8 \%$ in the $64 \mu \mathrm{g} / \mathrm{ml}, 98.3 \pm 2.7 \%$ in the $128 \mu \mathrm{g} / \mathrm{ml}$, and $97.9 \pm 2.3 \%$ in the $256 \mu \mathrm{g} / \mathrm{ml} \mathrm{rHMGN} 2$-treated cells compared with untreated cells, which was set at $100 \%$.

To determine if this rHMGN2-induced decrease of intracellular K. pneumoniae 03183 from T 24 cells involved a di-

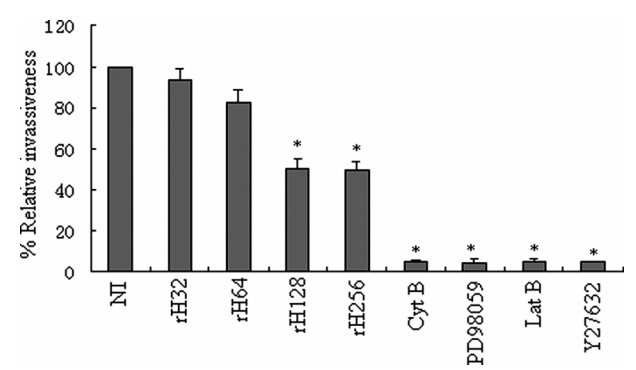

Fig. 1. Effects of Different Inhibitors on the Internalization of K. pneumoniae 03183 into Human Bladder Epithelial T 24 Cells

The inhibitors rHMGN2 $32 \mu \mathrm{g} / \mathrm{ml}$ (rH32), rHMGN2 $64 \mu \mathrm{g} / \mathrm{ml}$ (rH64), rHMGN2 $128 \mu \mathrm{g} / \mathrm{ml}(\mathrm{rH} 128), \mathrm{rHMGN} 2256 \mu \mathrm{g} / \mathrm{ml}(\mathrm{rH} 256)$, cytochalasin B $20 \mu \mathrm{M}$ (CytB) or PD98059 $20 \mu \mathrm{M}$ (PD) or Latrunculin B $5 \mu \mathrm{M}$ (Lat B) or Y-27632 $10 \mu \mathrm{M}$ were added $2 \mathrm{~h}$ before the addition of bacteria (see Materials and Methods). Invasion efficiency in the absence of inhibitors (NI) was defined as $100 \%$ relative invasiveness. Each column represents the average of at least three results in duplicate. $* p<0.05 v s$. NI.

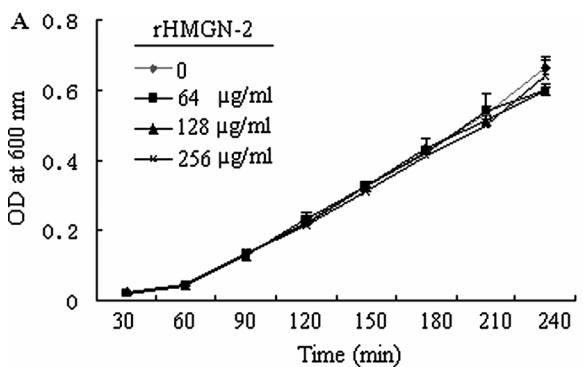

rect action of the protein on the $K$. pneumoniae, bacteria were exposed to rHMGN2 during growth in LB broth. rHMGN2 did not affect extracellular bacterial growth (Fig. 2A). To determine the antibacterial effects of rHMGN2 against $K$. pneumoniae 03183 , bacteria were incubated with different concentrations of rHMGN2 in PBS at $37^{\circ} \mathrm{C}$ for 0,2 and $4 \mathrm{~h}$. Bacteria incubated with all concentrations of rHMGN2 showed no antibacterial effects in a dose-dependent manner up to $4 \mathrm{~h}$ (Fig. 2B). In all of these assays, rHMGN2 exhibited no appreciable antibacterial activity against $K$. pneumoniae 03183 , suggesting that the rHMGN2induced decrease of intracellular bacteria was mediated indirectly via effects on host cells.

Signaling of rHMGN2 Inhibiting Invasion Activity of K. pneumoniae 03183 to T 24 Cells by MAPK Kinase Mitogen-activated protein kinase (MAPK) family members are involved in host cell invasion by several pathogenic bacteria such as Staphylococcus aureus (S. aureus), Chlamydia pneumoniae, Listeria monocytogenes (L. monocytogenes), Enteropathogenic Escherichia coli (EPEC) and Salmonella typhimurium (S. typhimurium). ${ }^{17-21)}$ Pretreatment with the MEK1/2 inhibitor PD98059 significantly reduced K. pneumoniae 03183 uptake by T24 cells (Fig. 1). The result demonstrated the essential role of MAPK for K. pneumoniae 03183 internalization into T 24 epithelial cells. As shown in Fig. 3A, K. pneumoniae 03183 stimulation increased ERK phosphorylation compared to unstimulated cells. To observe the effects of rHMGN2 on ERK phosphorylation, infected or uninfected cells were pretreated with rHMGN2 for various time points $(10,30,60,120,240 \mathrm{~min})$. rHMGN2 had a direct effect on ERK dephosphorylation in a time dependent manner (Fig. 3). These results suggested that rHMGN2 inhibited the invasion activity of $K$. pneumoniae 03183 into T 24 cells by interfering with ERK signaling.

Actin Cytoskeleton Changes after Pretreatment of T 24 Cells with rHMGN2 Most invasive bacteria enter eucaryotic cells via a microfilament-dependent process. ${ }^{22)}$ To examine the requirement for host cell actin cytoskeleton for $K$. pneumoniae 03183 internalization, T 24 cells were pretreated with $20 \mu \mathrm{M}$ Cyt B or $5 \mu \mathrm{M}$ Lat B. After the drug was washed out, the cells were incubated with $K$. pneumoniae 03183 for $2 \mathrm{~h}$ at $37^{\circ} \mathrm{C}$. Cytochalasin B disrupts actin filament integrity by capping the barbed end of actin. ${ }^{23)}$ Latrunculin B depolymerizes F-actin by preventing addition of G-actin monomers. ${ }^{24)}$ Treatment of T 24 cells with cytochalasin B or latrunculin B inhibited the internalization of K. pneumoniae 03183 (Fig. 1). The inhibitory effects of microfilament-dis-

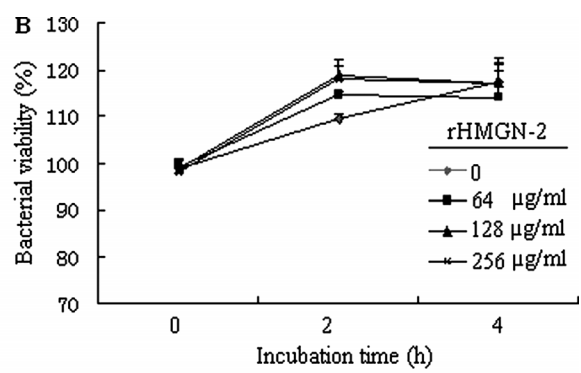

Fig. 2. rHMGN2 Does Not affect Extracellular Bacterial Growth or Bacterial Viability

(A) Effect of rHMGN2 on the growth of $K$. pneumoniae 03183 in LB broth. (B) Effect of rHMGN2 on K. pneumoniae 03183 viability. Bacterial viability was measured based on CFUs on culture plates, and the rate of bacterial viability was compared with rHMGN2-untreated PBS as a control. 

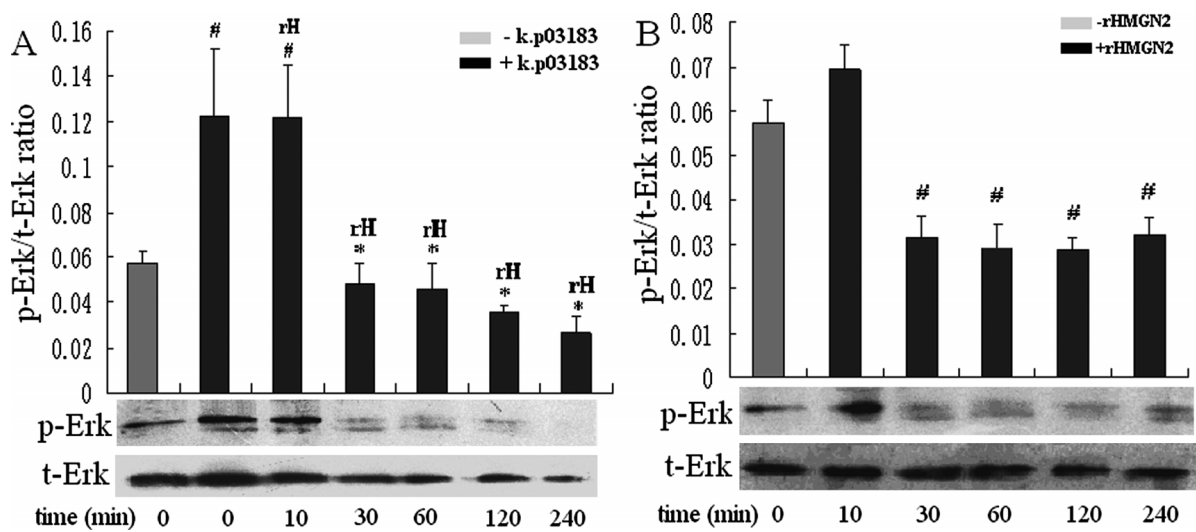

Fig. 3. ERK Activation

(A) Pretreatment infected cells with $128 \mu \mathrm{g} / \mathrm{ml} \mathrm{rHMGN} 2(\mathrm{rH})$; (B) uninfected cells were treated with $128 \mu \mathrm{g} / \mathrm{ml} \mathrm{rHMGN} 2$. Equal amounts of total cell lysates were subjected to SDS-PAGE. Immunoblotting was then performed using the indicated antibodies. $* p<0.05$ vs. with K. pneumoniae 03183 , \#p<0.05 vs. without K. pneumoniae 03183 (or $\mathrm{rH}$ ).
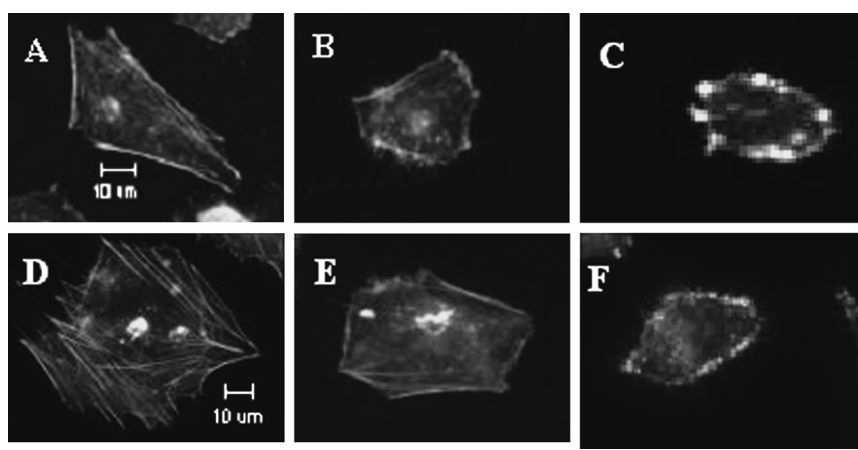

Fig. 4. Fluorescence Images of Cytoskeletal Actin in T 24 Cells

(A) Uninfected cells; (B) pretreatment uninfected cells with rHMGN2 for $2 \mathrm{~h}$; (C) pretreatment uninfected cells with $20 \mu \mathrm{M}$ cytochalasin B for $2 \mathrm{~h}$; (D) infected cells; (E) pretreatment infected cells with rHMGN2 for $2 \mathrm{~h}$; (F) pretreatment infected cells with $20 \mu \mathrm{M}$ cytochalasin $\mathrm{B}$ for $2 \mathrm{~h}$ after application of rhodamin-phalloidin to culture media for $30 \mathrm{~min}$.

rupting agents Cyt B and Lat B on T 24 cell invasion by $K$. pneumoniae indicated that the bacteria recruited actin polymerization for internalization.

To obtain direct morphological evidence, cells were preincubated with $128 \mu \mathrm{g} / \mathrm{ml}$ rHMGN2 or $20 \mu \mathrm{M}$ cytochalasin B for $2 \mathrm{~h}$, followed by stimulation with $K$. pneumoniae 03183 . After each indicated time, cells were stained with rhodaminphalloidin and analyzed by fluorescence microscopy. Phalloidins are known to interact with high affinity with filamentous F-actin but not with monomeric G-actin, and can therefore be used to quantitate the amount of polymerized actin in fixed cells. ${ }^{25)}$ As shown in Fig. 4, in uninfected cells, well-organized actin filaments were clear. K. pneumoniae 03183 induced actin polymerization. In contrast, exposure to cytochalasin B alone resulted in disruption of the filamentous actin structures. Pretreatment with rHMGN2 apparently eliminated the stress fibers and decreased the overall content of F-actin and most of the actin filaments were fragmented. These data clearly demonstrated the effect of rHMGN2 on actin filament disruption under the experimental conditions. To quantify this process, we repeated the experiment, and after incubation the samples were fixed in paraformaldehyde and analyzed by flow cytometry. The mean fluorescence detected for only infected cells was 4982 while the mean fluorescence was obviously decreased in the presence of rHMGN2 or cy-
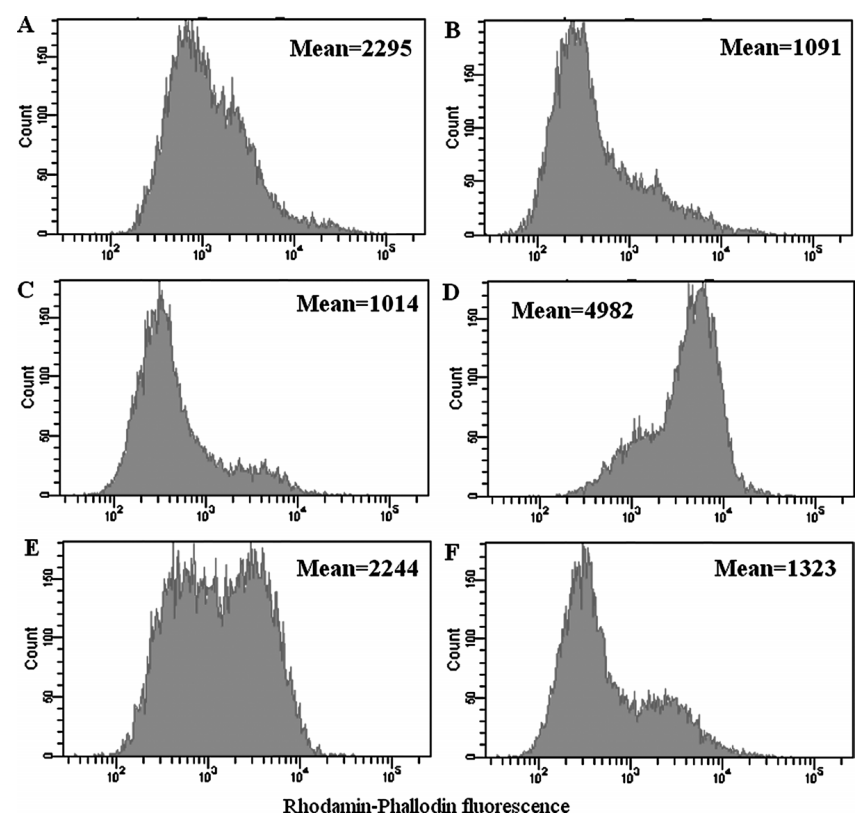

Fig. 5. Flow Cytometry Analysis of Cytoskeletal Actin in T 24 Cells

Histograms show the fluorescence intensity. (A) Uninfected cells; (B) pretreatment uninfected cells with rHMGN2 for $2 \mathrm{~h}$; (C) pretreatment uninfected cells with $20 \mu \mathrm{M}$ cytochalasin B for $2 \mathrm{~h}$; (D) infected cells; (E) pretreatment infected cells with rHMGN2 for $2 \mathrm{~h}$; (F) pretreatment infected cells with $20 \mu \mathrm{m}$ cytochalasin B for $2 \mathrm{~h}$ after application of rhodamin-phalloidin to culture media for $30 \mathrm{~min}$.

tochalasin B (2244 and 1323, respectively) (Fig. 5). The use of flow cytometry allowed us to extend our earlier observations that relied mostly on fluorescence microscopy, which showed that rHMGN2 can disassemble actin cytoskeleton.

Signaling of rHMGN2 Induced F-Actin Reorganization Mediated by Cofilin Kinase The cofilin protein appears to be a major factor contributing to actin depolymerization in cells, which is essential for actin filament dynamics and cytoskeleton reorganization. ${ }^{26-28)}$ To examine the role of cofilin in $K$. pneumoniae 03183 invasion of T 24 cells, monolayers were incubated with the Rho kinase inhibitor Y-27632 $(10 \mu \mathrm{M})$ in experimental medium for $2 \mathrm{~h}$ before addition of the bacteria and invasion assays were performed. Y-27632 exhibited inhibition of $K$. pneumoniae 03183 invasion of T 24 cells (Fig. 1). As shown in Fig. 6C, K. pneumoniae 03183 stimulation increased cofilin phosphorylation com- 

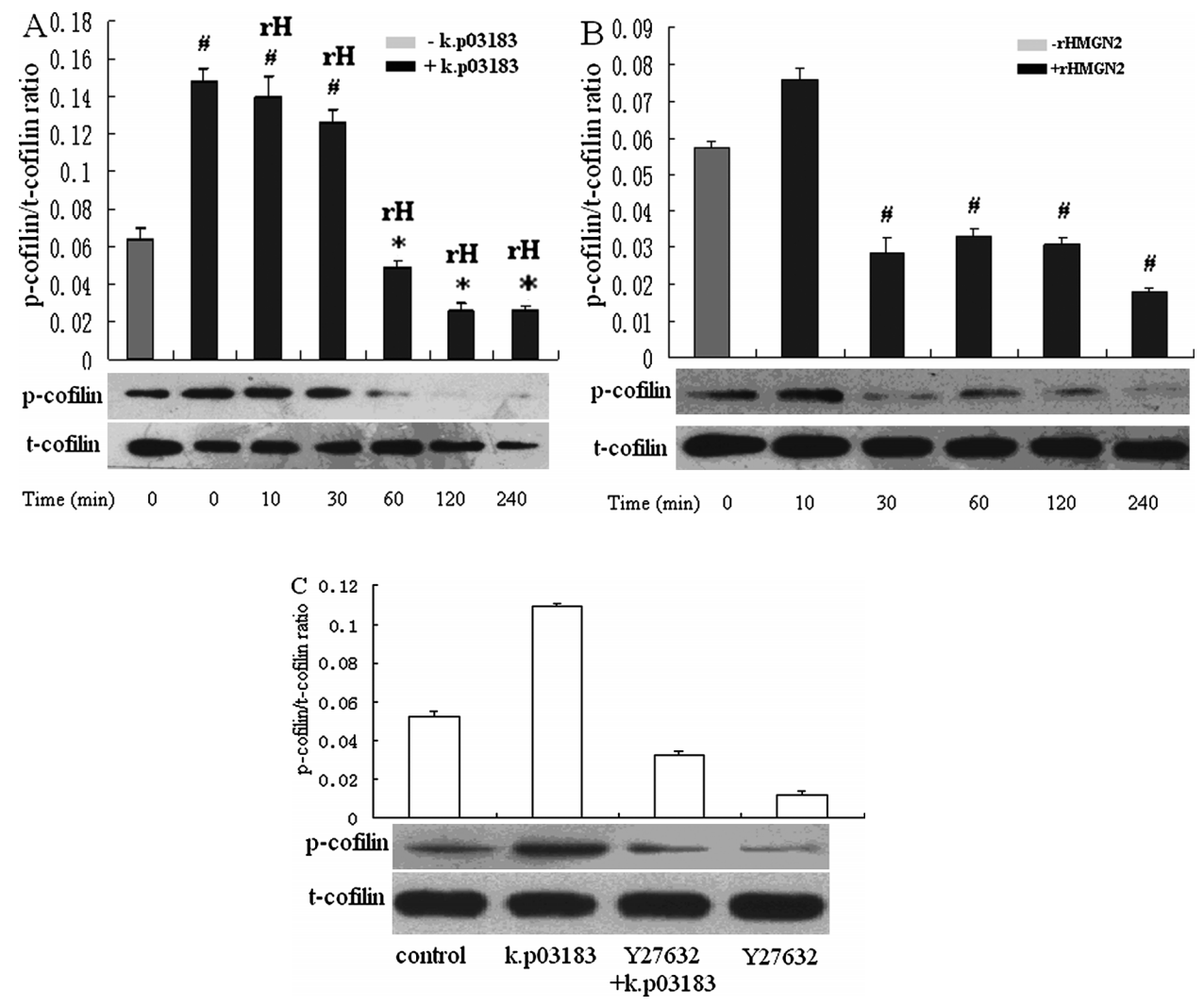

Fig. 6. Cofilin Activation

(A) Pretreatment infected T 24 cells with $128 \mu \mathrm{g} / \mathrm{ml} \mathrm{rHMGN} 2$ (rH); (B) uninfected cells were treated with $128 \mu \mathrm{g} / \mathrm{ml} \mathrm{rHMGN} 2$ for the indicated time points; (C) K. pneumoniae 03183 induced cofilin phosphorylation in the presence of Y-27632. Equal amounts of total cell lysates were subjected to SDS-PAGE. Immunoblotting was then performed using the indicated antibodies. $* p<0.05 v$ s. with $K$. pneumoniae $03183, \# p<0.05$ vs. without $K$. pneumoniae 03183 (or $\mathrm{rH}$ )

pared to unstimulated cells. Treatment with Y-27632 decreased levels of phosphorylated cofilin. In cells treated with Y-27632 followed by K. pneumoniae 03183 infection, cofilin phosphorylation was $55 \%$ of control levels. Taken together, these studies demonstrate K. pneumoniae 03183 invasion of T 24 cells requires cofilin phosphorylation. To observe the effects of rHMGN2 on cofilin phosphorylation, infected or uninfected cells were pretreated with rHMGN2 for various time points $(10,30,60,120,240 \mathrm{~min})$. The levels of cofilin phosphorylation pretreated with rHMGN2 were decreased in a time dependent manner (Figs. 6A, B). These results suggested that disruption of F-actin by rHMGN2 increased by interfering with cofilin signaling.

\section{DISCUSSION}

UTI is one of the most common bacterial infections that affect humans throughout their life span. Invasion into uroepithelial cells is a critical step in the ability of bacteria to cause UTI. ${ }^{29)}$ Our results show that pretreatment with HMGN2 significantly reduced $K$. pneumoniae 03183 uptake by T 24 cells. It induces these effects in the absence of host cell toxicity and without direct activity against the bacteria. This lack of a direct inhibitory effect on bacterial growth is particularly noteworthy, as it suggests that HMGN2 is less likely to promote microbial resistance than are conventional antibiotics.

Pathogens employ a variety of strategies to subvert and control normal host cellular functions. The ability to influ- ence key host intracellular-signaling molecules is a major advantage for many bacterial pathogens with respect to invasion and intracellular fate. ${ }^{30)}$ Mitogen-activated protein kinase (MAPK) family members are involved in host cell invasion by several pathogenic bacteria. ${ }^{17-21)}$ Invasion of epithelial cells represents a potential pathogenic mechanism for $K$. pneumoniae. We explored the role of mitogen-activated protein kinase kinases (MEK 1/2) and the extracellular signalregulated kinases (ERK 1/2) in K. pneumoniae invasion. Treatment of bladder epithelial cells with a highly specific inhibitor of MEK-1, PD98059, reduced K. pneumoniae invasion by approximately $90 \%$ without affecting bacterial association with the cells. We also found that $K$. pneumoniae 03183 activated ERK. In agreement with our results, $K$. pneumoniae infection can cause activation of $\mathrm{p} 44 / \mathrm{p} 42$ MAPK. ${ }^{31)}$ These data suggest that $K$. pneumoniae 03183 internalization by T 24 epithelial cells involves a pathway(s) that includes MEK and ERK signaling proteins. Pretreatment of infected cells with rHMGN2 greatly inhibited the ability of $K$. pneumoniae 03183 to invade T 24 bladder cells in a concentration-dependent fashion and prevented K. pneumoniae 03183-induced MAPK phosphorylation. These data indicated that MAPK may be involved in rHMGN2-mediated bacterial invasion.

Altering the host cytoskeleton is crucial for mediating pathogen adherence, invasion, and intracellular locomotion, such as Escherichia coli, Salmonella typhimurium, and Shigella flexneri. ${ }^{30)}$ Actin polymerization is essential for bacterial entry, ${ }^{32,33)}$ since treatment of cells by cytolchalasins ab- 
rogates it. ${ }^{32)}$ The cofilin protein appears to be a major factor contributing to actin depolymerization in cells, which is essential for actin filament dynamics and cytoskeleton reorganization. ${ }^{26-28)}$ Roles of cofilin in bacterial invasion of host cells have been reported. ${ }^{34,35)}$ The role of actin in the invasion process is investigated using a specific inhibitor of F-actin polymerization, cytochalasin B. T 24 bladder cells were incubated with cytochalasin $\mathrm{B}$ for $2 \mathrm{~h}$ prior to infection with K. pneumoniae 03183. Cytochalasin B dramatically reduced invasion ( $>95 \%$ inhibition), consistent with the results of Oelschlaeger and Tall. ${ }^{11)}$ In our study, K. pneumoniae 03183 enhanced cofilin phosphorylation and induced actin polymerization compared to uninfected cells while salmonella entry also requires activity cycles of host ADF and cofilin. ${ }^{34)}$ These data suggest that host cell actin polymerization may be play an important role in the $K$. pneumoniae 03183 invasion process by cofilin kinase. In the nucleus, HMGN proteins serve as a counterbalance to the action of the many ATP-dependent chromatin remodeling activities which are involved in the formation of actin filaments in the cytoplasm. ${ }^{5,36}$ Therefore, we speculated that HMGN2 may be associated with actin filaments. In our studies, we also found that rHMGN2 decreased basal and K. pneumoniae 03183-induced cofilin phosphorylation and inhibited $K$. pneumoniae 03183-induced actin polymerization. These results clearly demonstrate the effect of rHMGN2 on actin filament disruption under the experimental conditions by cofilin kinase.

In the present study, at $30 \mathrm{~min}$ after treatment with rHMGN2, the phosphorylation level of ERK by K. pneumoniae 03183 was decreased, but $K$. pneumoniae 03183induced phosphorylation of cofilin was not inhibited. At 60 min after treatment with rHMGN2, both $K$. pneumoniae 03183-induced phosphorylation of cofilin and ERK were decreased. This may indicate that ERK phosphorylation inhibited by rHMGN2 has no effect on cofilin dephosphorylation, which was partly supported by Won's findings, ${ }^{37)}$ but the suppressive effect of rHMGN2 on cofilin phosphoryation has an effect on ERK phosphorylation. The data can be interpreted as indicating that preconditioning with rHMGN2 inhibits cofilin phosphorylation, and then induces actin disruption which may block ERK phosphorylation. ${ }^{38-40)}$

Recent studies have shown that actin stress fiber formation was increased by inhibiting ERK phosphorylation, ${ }^{41)}$ and ERK dephosphorylation can result from inhibition of the cofilin kinase(s), which in turn induces actin depolymerization. In our experimental system, HMGN2 can not completely inhibit actin polymerization. The data indicate that actin depolymerization through cofilin dephosphorylation was too strong for actin stress fiber formation by inhibiting ERK phosphorylation.

Taken together, preconditioning with rHMGN2 inhibits cofilin phosphorylation, and then induces actin disruption which may block ERK phosphorylation. These changes lead to inhibit $K$. pneumoniae 03183 invasion of T 24 bladder epithelial cells and may provide insight into the development of more efficacious therapies for the treatment and prevention of UTI.

Acknowledgments China National Nature Science Fund 30671963 and China Medical Board of New York INC98681 .

\section{REFERENCES}

1) Grüneberg R.N., J. Antimicrob. Chemother, 33 (Suppl. A), 1-8 (2000).

2) Filozov A., Visintainer P., Carbonaro C., Aguero-Rosenfeld M., Wormser G. P., Montecalvo M. A., Am. J. Infect. Control, 37, 723728 (2009)

3) Shirakawa H., Herrera J. E., Bustin M., Postnikov Y., J. Biol. Chem., 275, 37937-37944 (2000).

4) Subramanian M., Gonzalez R. W., Patil H., Ueda T., Lim J. H., Kraemer K. H., Bustin M., Bergel M., FEBS J., 276, 6646-6657 (2009).

5) Rattner B. P., Yusufzai T., Kadonaga J. T., Mol. Cell, 34, 620-626 (2009).

6) Amen M., Espinoza H. M., Cox C., Liang X., Wang J., Link T. M., Brennan R. G., Martin J. F., Amendt B. A., Nucleic Acids Res., 36, 462-476 (2008).

7) Feng Y., Huang N., Wu Q., Wang B., J. Leukoc. Biol., 78, 1136-1141 (2005).

8) Feng Y., Huang N., Wu Q., Bao L., Wang B. Y., Acta Pharmacol. Sin., 26, $1087-1092$ (2005).

9) Feng Y., He F., Zhang P., Wu Q., Huang N., Tang H., Kong X., Li Y., Lu J., Chen Q., Wang B., Antiviral Res., 81, 277-282 (2009).

10) Xiong W. B., Huang N., Feng Y., Wu Q., Wang B. Y., Chin. Med. J. (England), 121, 82-85 (2008).

11) Oelschlaeger T. A., Tall B. D., Infect. Immun., 65, 2950-2958 (1997).

12) Vongsa R. A., Zimmerman N. P., Dwinell M. B., J. Biol. Chem., 284, $10034-10045$ (2009).

13) Weiner D. J., Bucki R., Janmey P. A., Am. J. Respir. Cell Mol. Biol., 28, 738-745 (2003).

14) Ohtake T., Fujimoto Y., Ikuta K., Saito H., Ohhira M., Ono M., Kohgo Y., Br. J. Cancer, 81, 393-403 (1999).

15) Degryse B., Bonaldi T., Scaffidi P., Müller S., Resnati M., Sanvito F., Arrigoni G., Bianchi M. E., J. Cell Biol., 152, 1197-1206 (2001).

16) Kihlström E., Andåker L., J. Antimicrob. Chemother, 15, 723-728 (1985).

17) Coombes B. K., Mahony J. B., Cell. Microbiol., 4, 447-460 (2002).

18) Czerucka D., Dahan S., Mograbi B., Rossi B., Rampal P., Infect. Immun., 69, 1298-1305 (2001).

19) Ellington J. K., Elhofy A., Bost K. L., Hudson M. C., Infect. Immun., 69, 5235-5242 (2001)

20) Hobbie S., Chen L. M., Davis R. J., Galán J. E., J. Immunol., 159, $5550-5559$ (1997)

21) Tang P., Sutherland C. L., Gold M. R., Finlay B. B., Infect. Immun., 66, 1106-1112 (1998)

22) Moulder J. W., Microbiol. Rev., 49, 298-337 (1985).

23) Cooper J. A., J. Cell Biol., 105, 1473-1478 (1987).

24) Zito K., Knott G., Shepherd G. M., Shenolikar S., Svoboda K., Neuron, 44, 321-334 (2004).

25) Knowles G. C., McCulloch C. A., J. Histochem. Cytochem., 40, 1605-1612 (1992)

26) Pavlov D., Muhlrad A., Cooper J., Wear M., Reisler E., J. Mol. Biol., 365, 1350-1358 (2007).

27) Nebl G., Fischer S., Penzel R., Samstag Y., Cell. Signal., 16, 235-243 (2004).

28) McGough A., Pope B., Chiu W., Weeds A., J. Cell Biol., 138, 771781 (1997).

29) Tsai K. W., Lai H. T., Tsai T. C., Wu Y. C., Yang Y. T., Chen K. Y., Chen C. M., Li Y. S., Chen C. N., J. Biomed. Sci., 16, 91-104 (2009).

30) Bhavsar A. P., Guttman J. A., Finlay B. B., Nature (London), 449, $827-834$ (2007).

31) Wu J. H., Hong L. C., Tsai Y. Y., Chen H. W., Chen W. X., Wu T. S., Cell. Microbiol., 8, 1467-1474 (2006).

32) Sansonetti P. J., Tran Van Nhieu G., Egile C., Clin. Infect. Dis., 28, $466-475$ (1999).

33) Rottner K., Stradal T. E., Wehland J., Dev. Cell, 9, 3-17 (2005).

34) Dai S., Sarmiere P. D., Wiggan O., Bamburg J. R., Zhou D., Cell. Microbiol., 6, 459-471 (2004).

35) Bierne H., Gouin E., Roux P., Caroni P., Yin H. L., Cossart P., J. Cell Biol., 155, 101-112 (2001).

36) Farrants A. K., FEBS Lett., 582, 2041-2050 (2008).

37) Won K. J., Park S. H., Park T., Lee C. K., Lee H. M., Choi W. S., Kim S. J., Park P. J., Jang H. K., Kim S. H., Kim B., J. Pharmacol. Sci., 
108, 372-379 (2008).

38) Zhou S., Bachem M. G., Seufferlein T., Li Y., Gross H. J., Schmelz A., Cell. Signal., 20, 695-704 (2008).

39) Yang C., Patel K., Harding P., Sorokin A., Glass W. F. 2nd, Exp. Cell Res., 313, 1240-1250 (2007).
40) Huang F., Chotiner J. K., Steward O., J. Neurosci., 27, 9054-9067 (2007).

41) Klein R. M., Spofford L. S., Abel E. V., Ortiz A., Aplin A. E., Mol. Biol. Cell, 19, 498-508 (2008). 